\title{
ANÁLISIS DEL ESPESOR DE LA CAPA DE FIBRAS NERVIOSAS EN NIÑOS DE 6 A 9 AÑOS
}

\section{RETINAL NERVE FIBER LAYER THICKNESS ANALYSIS IN CHILDREN FROM 6 TO 9 YEARS OF AGE}

COLOMA-GONZÁLEZ I I , GARCÍA-CONCA V , MENGUAL-VERDÚ E², HUESO-ABANCÉNS JR ${ }^{3}$

\section{RESUMEN}

Objetivo: Establecer una base de datos para el GDx-VCC representativa para nuestra población de entre 6 a 9 años y analizar los datos obtenidos por rango de edades.

Método: Se analizó la densidad de la CFN mediante oftalmoscopía de barrido con láser (GDx) a 116 niños procedentes de un único colegio y los resultados fueron sometidos a estudio estadístico por edades y en global a fin de determinar el rango de valores considerable como la normalidad para esa población y las posibles diferencias entre ellos.

Resultados: En el análisis global por cuadrantes de los datos estudiados por el GDx se encontró un promedio TSNIT de 59,43 (IC95\% 58,41-60,45); superior de 71,35 (IC95\% 69,99-72,70); inferior de 70,08 (IC95\% 68,71-71,45) y una desviación estándar TSNIT de 25,11 (IC95\% 24,36-25,86). En el

\begin{abstract}
Purpose: To establish a database of scanning laser polarimetry (GDx) parameters for children between 6 and 9 years of age and compare the results at each age. Methods: The retinal nerve layer thickness of 116 children from one school was evaluated with GDx and the results were analyzed for each age and for the entire group to determinate the normal range for that population.

Results: In the global analysis of the software-derived GDx parameters, we obtained a TSNIT average of 59.43 (IC95\% 58.41-60.45), a superior average of 71.35 (IC95\% 69.99-72.70), an inferior average of 70.08 (IC95\% 68.71-71.45), and a TSNIT Std. Deviation of 25.11 (IC95\% 24.36-25.86). The results from the analysis for age were similar to the global results.
\end{abstract}

Conclusions: Given the low level of cooperation required, GDx can be used without problem to

\footnotetext{
Recibido: 28/9/06. Aceptado: 17/9/07.

Hospital Universitario San Juan de Alicante. Alicante. España.

1 Licenciado en Medicina.

2 Doctor en Medicina. Jefe de Sección.

3 Doctor en Medicina. Jefe de Servicio.

Comunicación presentada parcialmente en el LXXXII Congreso de la S.E.O. (La Coruña 2006).

Correspondencia:

Itziar Coloma González

Hospital Universitario San Juan de Alicante

Ctra. N-332 Alicante-Valencia, s/n

03550 Alicante

España

E-mail: icg_ct@yahoo.es
} 
análisis por edades se observan valores medios por cuadrantes comparables a los del global.

Conclusiones: El estudio de la CFN mediante GDx se puede realizar sin problema en la población infantil de 6 a 9 años dada la mínima colaboración requerida, obteniendo así datos objetivos sobre su estado y desarrollo durante estas edades.

Palabras clave: Polarimetría láser, GDx VCC, espesor de la capa de fibras nerviosas, glaucoma infantil, sujetos normales. study the retinal nerve fiber layer in 6-9-year-old children. GDx could provide objective information about the state of development of the retinal nerve fiber layer during this period of life (Arch Soc Esp Oftalmol 2007; 82: 705-710).

Key words: Scanning laser polarimetry, GDx VCC, retinal nerve fiber layer thickness, childhood glaucoma, normal subjects.

\section{INTRODUCCIÓN}

La oftalmoscopía por barrido con láser (GDx-VCC) permite el estudio del estado de la capa de fibras nerviosas de la retina (CFNR) por la técnica de escaneo láser polarimétrico con compensación variable de la córnea mediante el empleo de un láser de diodo de baja intensidad y $780 \mathrm{~nm}$ de longitud de onda.

El aparato emite un haz de láser que cambia de estado al pasar por el polarizador, proyectando dos haces perpendiculares hacia la CFNR. El cálculo del espesor de la CFNR es posible gracias a sus propiedades polarizantes, siendo directamente proporcional a la modificación de su estado de polarización o retraso sufrido por el haz que viaja en paralelo a la CFNR (1). Además, gracias al sistema de compensación variable de la córnea (VCC) determina y corrige la birrefringencia del segmento anterior anulando su efecto en el resultado. De esta manera se obtiene una medida cuantitativa y reproducible de su estado mediante una prueba no invasiva, indolora y que no precisa de instilación de gotas. El aparato tarda aproximadamente un segundo en realizar la medida, requiere una colaboración mínima por parte del paciente y permite el estudio secuencial o seguimiento del mismo detectando pequeños cambios difícilmente objetivables de otra manera. Aspectos todos ellos que cobran aún mayor importancia al hablar de pacientes en edad pediátrica. Por ello, consideramos que podría tratarse de una prueba con características óptimas para ser utilizada en la población infantil y llegar a ser un gran apoyo en la evaluación de este tipo de pacientes.

El objetivo principal de este estudio es establecer una base de datos para el GDx-VCC representativa para nuestra población de entre 6 y 9 años que sirva de apoyo para futuras líneas de investigación sobre la aplicación de esta técnica en el glaucoma pediátrico. Y, en segundo lugar, analizar los resultados por rangos de edades y valorar de manera objetiva su aplicabilidad en la población infantil.

\section{SUJETOS, MATERIAL Y MÉTODOS}

Para ello se realizó el estudio de la capa de fibras nerviosas de la retina (CFNR) en un total de 116 niños de entre 6 y 9 años, procedentes todos ellos del único colegio de enseñanza primaria sito en Biar (Alicante). Los datos descriptivos de distribución de nuestra población a estudio se muestran en la tabla I.

Se excluyeron todos aquellos sujetos y/u ojos con ambliopía, ametropía no refractable (AVCC $\leq 0,8$ ), estrabismo, cirugía o enfermedad ocular previa con secuelas permanentes, uso de colirios (mióticos, corticoides...) en la semana previa y antecedentes familiares (de primer grado) de glaucoma.

Se utilizaron dos analizadores de la capa de fibras nerviosas GDx-VCC (NFA, Laser Diagnostic Technologies Inc, San Diego, CA, USA) que permiten obtener medidas «in vivo» del espesor de la CFNR (fig. 1). Este instrumento posee una fuente emisora de láser diodo, un polarizador, un modulador de la polarización, un compensador de la polarización corneal y una unidad de detección.

Las imágenes se obtuvieron por tres médicos residentes con experiencia en la prueba mayor de

Tabla I. Descriptiva de la población de estudio

\begin{tabular}{lllllll}
\hline & 6 años & 7 años & 8 años & 9 años & total & $\%$ total \\
\hline Mujeres & 15 & 11 & 13 & 15 & 54 & 46,5 \\
Hombres & 17 & 15 & 12 & 18 & 62 & 53,5 \\
Global & 32 & 26 & 25 & 33 & 116 & 100 \\
\%total & 27,6 & 22,4 & 21,5 & 28,5 & & \\
\hline \hline
\end{tabular}




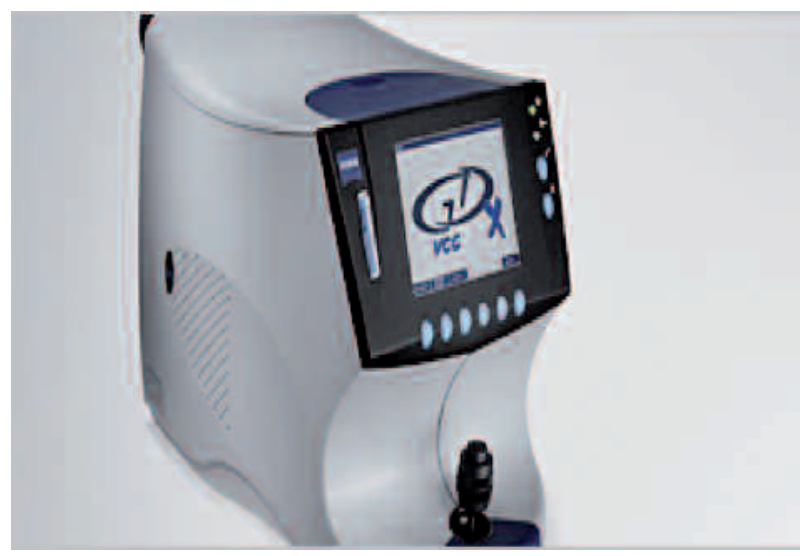

Fig. 1: Analizador de la capa de fibras nerviosas (GDX$V C C)$.

dos años. Cada niño fue examinado indistintamente por uno de los tres exploradores empleando los parámetros estándares del GDx.

Para la medida del espesor de la CFNR se utilizó un óvalo de 10 pixeles de ancho, centrado sobre la papila, situado a 1,75 diámetros de la papila y dividido en cuatro sectores estándares del GDx (temporal $155^{\circ}-205^{\circ}$, superior $35^{\circ}-155^{\circ}$, nasal $325^{\circ}-35^{\circ}$ e inferior $205^{\circ}-325^{\circ}$ ). De los datos suministrados por el aparato se analizaron:

— Espesor medio y desviación estándar total (óvalo de medida).

- Espesor medio del cuadrante superior.

- Espesor medio del cuadrante inferior.

- Simetría interocular.

- NFI.

Se analizaron estas cinco variables en global y por rango de edades (6-9). Para el análisis de normalidad se empleó el test de Kolmogorov-Smirnov. Expresamos los resultados con medidas descriptivas de tendencia central, media e intervalo de confianza al 95\%. Para el análisis comparativo entre los distintos grupos de edad se empleó el test de ANOVA. Se consideró como diferencia estadísticamente significativa aquellos valores de $\mathrm{p}<0,05$. El test de la T-Student para muestras independientes se utilizó para la comparación entre dos grupos, de manera que nos permite determinar dónde están las diferencias estadísticamente significativas detectadas por ANOVA.

Para valorar si la prueba es realizable en este grupo de edad se estudió el porcentaje de niños en los que se pudo realizar sin problemas.

El estudio estadístico se realizó con el programa SPSS, versión 12.0 (SPSS Inc,Chicago, Illinois, EEUU).

\section{RESULTADOS}

Para el estudio estadístico se emplearon 217 ojos (116 sujetos) de un total de 231, excluyéndose el resto según los criterios descritos en el apartado anterior o por falta de colaboración durante la realización de la prueba.

Los datos observados siguen una distribución normal. En la tabla II se presentan los resultados obtenidos en el análisis global de los espesores medios recogidos por el GDx y en la tabla III los del análisis por edades. No se encontraron diferencias significativas entre los distintos rangos de edad al comparar los valores de espesor medio y desviación estándar total, espesor medio del cuadrante superior, espesor medio del cuadrante inferior (tabla IV).

También se recogieron y analizaron la simetría inter-ocular y el NFI (tabla V), encontrando una diferencia clínicamente significativa al comparar la

Tabla II. Espesores medios de la capa de fibras nerviosas globales

\begin{tabular}{lcc}
\hline & Media & Interv. Confianza 95\% \\
\hline Promedio TSNIT & 59,43 & $85,41-60,45$ \\
Promedio Superior & 71,35 & $69,99-72,70$ \\
Promedio Inferior & 70,08 & $68,71-71,45$ \\
Desviación Estándar & 25,11 & $24,36-25,86$ \\
\hline \hline
\end{tabular}

Tabla III. Espesores medios de la capa de fibras nerviosas por edades

\begin{tabular}{|c|c|c|c|c|c|c|c|c|}
\hline & \multicolumn{2}{|c|}{6 años } & \multicolumn{2}{|c|}{7 años } & \multicolumn{2}{|c|}{8 años } & \multicolumn{2}{|c|}{9 años } \\
\hline & MEDIA & IC $95 \%$ & MEDIA & IC $95 \%$ & MEDIA & IC $95 \%$ & MEDIA & IC $95 \%$ \\
\hline Promedio TSNIT & 59,32 & $57,79-60,85$ & 58,97 & $56,71-61,23$ & 61,18 & $57,99-64,37$ & 58,53 & $57,00-60,05$ \\
\hline Promedio Superior & 70,73 & $68,41-73,04$ & 71,50 & $68,82-74,18$ & 73,58 & $64,6971,28$ & 70,12 & $67,76-72,5$ \\
\hline Promedio Inferior & 71,32 & $68,98-73,65$ & 68,11 & $64,94-71,28$ & 70,95 & $67,73-74,15$ & 69,58 & $66,89-72,27$ \\
\hline Desviación Estándar TSNIT & 25,31 & $23,95-26,66$ & 24,55 & $22,89-26,21$ & 25,24 & $23,56-26,91$ & 25,26 & $23,72-26,79$ \\
\hline
\end{tabular}


Tabla IV. Significación clínica del análisis comparativo de los espesores medios de la CFNR mediante el test de la t-Student

\begin{tabular}{lcccccc}
\hline & 6 vs 7 & 6 vs 8 & 6 vs 9 & 7 vs 8 & 7 vs 9 & $0,0,737$ \\
\hline Promedio TSNIT & 0,789 & 0,250 & 0,465 & 0,255 & 0,111 \\
Promedio Superior & 0,665 & 0,182 & 0,714 & 0,373 & 0,439 & 0,114 \\
Promedio Inferior & 0,097 & 0,846 & 0,329 & 0,209 & 0,476 & 0,511 \\
Desviación Estándar & 0,476 & 0,949 & 0,963 & 0,557 & 0,530 \\
\hline \hline
\end{tabular}

Tabla V. Estudio de la simetría inter-ocular y NFI por edades

\begin{tabular}{lccccc}
\hline & 9 años & 8 años & 7 años & 6 años & Total \\
\hline Simetría IO* & 0,83 & 0,80 & 0,60 & 0,74 & 0,74 \\
NFI & $(28)$ & $(21)$ & $(22)$ & $(32)$ & $(103)$ \\
\hline \hline
\end{tabular}

* Entre paréntesis el número de pacientes.

simetría inter-ocular media (ANOVA $\mathrm{p}=0,003$ ). Mediante la t-Student observamos que la diferencia se encontraba al comparar el grupo de los 7 años con el resto ( 7 vs $6 \mathrm{p}=0,001 ; 7$ vs $8 \mathrm{p}=0,25 ; 7$ vs 9 $\mathrm{p}=0,003$ ).

Se realizó la prueba en el 93,1\% de los niños incluidos a priori en el estudio y en el $96,5 \%$ de los niños que cumplían los criterios de inclusión. Tres niños se excluyeron por no cumplir los criterios de inclusión $(3,4 \%)$ y en cuatro no se consiguió realizar la exploración con éxito (dos de 6 años, uno de 7 años y uno de 9 años) por falta de colaboración $(3,5 \%)$.

\section{DISCUSIÓN}

En este trabajo hemos presentado una base de datos para el GDx-VCC representativa de nuestra población entre 6 y 9 años ya que la base proporcionada por el fabricante no incluye sujetos por debajo de los 18 años y en la literatura tampoco encontramos descripciones del espesor de la CFNR en la población menor de 15 años $(2,3)$. Nuestra intención es abrir las puertas a futuras líneas de investigación en el campo del glaucoma pediátrico. También se presenta un estudio estadístico de los datos recogidos con el fin de valorar posibles diferencias entre los grupos de edad estudiados. Así, hemos comprobado que no existen diferencias para los espesores de la CFN entre ellos ni tampoco para los valores del NFI pero sí en la simetría IO. Encontramos una diferencia clínicamente significativa entre los niños de 7 años y el resto de los grupos. Esto podría deberse a un desarrollo más asimétrico de la retina durante esta época o a un sesgo de selección.

Al comparar nuestros resultados con los encontrados en la literatura, vemos que los valores presentados para la población adulta son algo inferiores a los recogidos en nuestro estudio mientras que los presentados para la población infantil tienden a ser superiores. Así, por ejemplo, Capriali et al. reportan valores de 55,1 DE 5,9 para el promedio TSNIT; de 65,9 DE 7,9 para el promedio superior; de 64,8 DE 8,7 para el promedio inferior; y de 21,3 DE 4,7 para la desviación estándar del TSNIT (4). Mientras que los valores reportados por Vijaya et al. son de $62,24 \mathrm{DE} 10,44$ para el promedio TSNIT; de 76,71 DE 10,22 para el promedio superior y de $79,9 \mathrm{DE} 12,16$ para el promedio inferior. La explicación a este hecho sería la pérdida axonal progresiva con la edad descrita ya por otros autores (3) en el primer caso y diferencias en la etnia de la población a estudio en el segundo (5).

La colaboración de los niños fue muy buena, con un alto porcentaje de pruebas realizadas. Lo que nos indica que es una prueba apta para este grupo de edad y, por lo tanto, que podría ser útil en el manejo de la patología pediátrica de nervio óptico y CFNR al igual que lo es en la del adulto. Por ejemplo, en el glaucoma pediátrico. Ésta es una entidad poco frecuente que, por lo general, acompaña a otras enfermedades también relativamente raras. Estudios europeos reportan una incidencia de 1:12500 recién nacidos y es responsable de aproximadamente el 4\% de los casos de ceguera en Europa occidental (6). De las múltiples clasificaciones que existen $(6,7)$ nosotros preferimos la de Hoskins, que lo divide en: congénito, con aumento de la presión intrauterinamente manifestándose ya al nacimiento (40\%); infantil, que se manifiesta entre el $1 .^{\text {er }}$ mes y antes de los 2 años (55\%); y juvenil, con elevación de la presión después de los 2 años pero antes de los 16. De entre todos ellos, el más frecuente es el glaucoma congénito primario. 
El diagnóstico de sospecha es clínico y para confirmarlo se precisa de toma de la presión intraocular, exploración del polo anterior (oftalmoscopía, gonioscopía y medición de diámetros corneales) y excavación papilar. Estas pruebas se suelen realizar bajo anestesia dada la corta edad de los pacientes.

Como el glaucoma del adulto, se trata de una enfermedad progresiva, con consecuencias no reversibles, que en muchos casos no muestra síntomas hasta estadios avanzados con pérdida visual importante. Su manejo es esencialmente quirúrgico con tratamiento médico como coadyuvante y los resultados visuales dependen directamente de la rapidez en instaurarse dicho tratamiento (6). Sin embargo, las técnicas diagnósticas convencionales precisan de una gran experiencia y largo aprendizaje si lo que se pretende es detectar estadios precoces de la enfermedad. Por ello, se han desarrollado nuevas técnicas basadas en la tecnología láser y óptica coherente cuyo objetivo es precisamente éste, el diagnóstico precoz del glaucoma. Entre ellas encontramos la oftalmoscopía o polarimetría láser de barrido (GDx-VCC) que permite una medida objetiva del espesor de la CFNR.

En conclusión, consideramos que es una prueba apta para la población infantil lo que abre un amplio campo de posibles indicaciones. Desde su posible aplicación para el diagnóstico precoz y seguimiento en el glaucoma infantil hasta aquellas nuevas indicaciones que están apareciendo en estos últimos años para la patología no glaucomatosa del nervio óptico y la CFNR (8-10).

\section{BIBLIOGRAFÍA}

1. García-Feijoo J, García-Sánchez J. Correlación entre el espesor de la capa de fibras nerviosas y el campo visual. Arch Soc Canar Oftal 2001; 12: 3-9.

2. Baquero Aranda IM, Morillo Sánchez MJ, García Campos JM. Estudio de parámetros de normalidad en relación a la edad con el tomógrafo óptico de coherencia. Arch Soc Esp Oftalmol 2005; 80: 225-232.

3. Toprak AB, Yilmaz OF. Relation of optic disc topography and age to thickness of retinal nerve fibre layer as measured using scanning laser polarimetry, in normal subjects. Br J Ophthalmol 2000; 84: 473-478.

4. Caprioli J, Nuori-Mahdavi K, Law SK, Badala F. Optic disc imaging in perimetrically normal eyes of glaucoma patients with unilateral field loss.Trans Am Ophthalmol Soc 2006; 104: 202-211.

5. Vijaya L, Varma M, Krishhna Kumar $R$, Saraniya AS, Babu $G$, Subash $S$, et al. Retinal nerve fiber layer measurements in indian eyes using the scanning laser polarimeter, GDx. Indian J Ophthalmol 2004; 52: 215-220.

6. Montezuma SR, Serrano-Camacho JC. Glaucoma Pediátrico en la Fundación Oftalmológica de Santander. Medunav 1999; 2. Disponible en URL: http://editorial. unab.edu.co/revistas/medunav/pdfs/r24ao_r1.html. Consultado el 29 de Junio 2006.

7. Walton DS. Primary congenital open angle glaucoma: a study of the anterior segment abnormalities. Trans Am Ophthalmol Soc 1979; 77: 746-768.

8. García CA, de Oliveira AG, de Lima CE, Rocha F, García Filho CA. Retinal nerve fiber layer análisis using GDx in 49 patients with chronic phase DUSN. Arq Bras Oftalmol 2006; 69: 631-635.

9. Sadun AA, Salomao SR, Berezovsky A, Sadun F, Denegri AM, Quiros PA, et al. Subclinical carriers and conversions in Leber hereditary optic neuropathy: a prospective psychophysical study. Trans Am Ophthalmol Soc 2006; 104: 51-61.

10. Foroozan R, Buono LM, Savino PJ, Sergott RC. Scanning laser polarimetry of the retinal nerve fiber layer in central retinal artery occlusion. Ophthalmology 2003; 110: 715-718. 